\title{
Florence Plet-Nicolas, La Création du Monde. Les noms propres dans le roman de Tristan en prose
}

\section{Richard Trachsler}

\section{(2) OpenEdition}

1 Journals

\section{Édition électronique}

URL : http://journals.openedition.org/studifrancesi/5846

DOI : 10.4000/studifrancesi.5846

ISSN : 2427-5856

Éditeur

Rosenberg \& Sellier

\section{Édition imprimée}

Date de publication : 1 mai 2011

Pagination : 143-144

ISSN : 0039-2944

\section{Référence électronique}

Richard Trachsler, «Florence Plet-Nicolas, La Création du Monde. Les noms propres dans le roman de Tristan en prose », Studi Francesi [En ligne], 163 (LV | I) | 2011, mis en ligne le 30 novembre 2015, consulté le 09 janvier 2021. URL : http://journals.openedition.org/studifrancesi/5846 ; DOI : https:// doi.org/10.4000/studifrancesi.5846

Ce document a été généré automatiquement le 9 janvier 2021.

\section{(c) $($ ) $\odot$}

Studi Francesi è distribuita con Licenza Creative Commons Attribuzione - Non commerciale - Non opere derivate 4.0 Internazionale. 


\title{
Florence Plet-Nicolas, La Création du Monde. Les noms propres dans le roman de Tristan en prose
}

\author{
Richard Trachsler
}

\section{RÉFÉRENCE}

FLORENCE PLET-NICOLAS, La Création du Monde. Les noms propres dans le roman de Tristan en prose, Paris, Champion, 2007 («Nouvelle Bibliothèque du Moyen Âge», 84), pp. 512.

1 La littérature arthurienne est marquée, voire définie, par la récurrence d'un certain nombre de personnages et lieux: Arthur, Guenièvre, Tristan, Yseult, Lancelot qui traversent Logres, Camaalot, Cardigan ou Corbenic. Certains de ces noms sont repris à une tradition antérieure - en général celtique-, mais d'autres sont créés par les romanciers français, qui constituent ainsi un univers cohérent, soutenu par une onomastique propre et «plausible». Le but du livre de Florence Plet est d'examiner ces noms propres et leur fonctionnement dans le Tristan en prose, la plus prolifique des sommes arthuriennes. Il ne s'agit pas d'un travail d'onomastique au sens linguistique, qui tournerait vite à vide, une fois établie la liste des toponymes réels et la façon dont sont créées, sur cette base, les villes et contrées imaginaires peuplées par des personnages eux aussi porteurs de noms vaguement celtisants. Il s'agit au contraire d'un travail d'onomastique littéraire, qui étudie, par exemple, la manière de désigner les personnages, la façon dont ils s'apostrophent, les effets de sens produits par l'anonymat, puis par la découverte du nom etc. C'est seulement dans sa dernière partie que Florence Plet s'autorise quelques spéculations isidoriennes sur ce qu'a pu évoquer telle ou telle sonorité pour un lecteur médiéval.

Le travail est organisé en quatre parties: dans un premier mouvement (Cerner le Nom propre, pp.17-152), on s'interroge sur le statut du nom propre médiéval en recourant aux travaux des logiciens et linguistes modernes. Comme aujourd'hui, le nom propre 
médiéval ne fonctionne pas de la même façon qu'un nom «ordinaire» du point de vue de la morphologie et de la syntaxe, où il reste difficile à cerner, et se singularise au niveau sémantique, particulièrement exploité par le romancier par le biais de toponymes composés comme Chastel Felon et d'anthroponymes en périphrase, comme Breüs sans Pitié. L'onomastique tristanienne partage avec l'onomastique réelle quelques traits, mais accuse surtout l'influence de la tradition littéraire antérieure qui, des Tristan en vers au Lancelot en prose, lui fournit le stock et le moule pour la sienne. F. Plet montre ainsi, passages à l'appui, que les noms celtiques étaient déjà devenus "aveugles» pour le public français du XIII ${ }^{e}$ siècle: le nom Tristan est rattaché à «triste», Marc s'appelle ainsi parce qu'il est né un mardi au mois de mars, etc. Les précisions «étymologiques» que contient le roman sont toutefois rares et invitent donc le critique moderne à privilégier d'autres approches et à poursuivre son enquête en se plongeant autrement dans cette déferlante de personnages nommés dont certains «auraient tout aussi bien pu rester anonymes» (p. 99). Par conséquent, dans un deuxième mouvement (La Quête du Nom, pp.153-253), Florence Plet s'attache à mettre en évidence l'importance du nom pour l'intrigue romanesque. Il existe tout un rituel régissant la requête de l'identité que l'on adresse à un inconnu, de même que le roman utilise des séquences standardisées pour l'identification et la reconnaissance d'un personnage anonyme. C'est précisément la variation qui intervient, dans le Tristan en prose, dans l'emploi de ces séquences traditionnelles depuis un demi-siècle qui fait la spécificité du roman en prose. Ce jeu autour de la connaissance, reconnaissance et méconnaissance «permet [...] à un roman de si longue haleine de se déployer sans s'effondrer malgré la longueur» (p. 253). La troisième partie (Le Déchiffrage du Nom, pp. 255-370) observe comment le roman en prose insère le nom propre dans le lignage ou dans l'espace. Le plus souvent, un personnage est ancré dans un lignage par rapport à un ancêtre, plus rarement un frère, voire un descendant. Souvent, toutefois, le renvoi ne renvoie à rien puisque le point de repère n'est pas identifiable en dehors du renvoi lui-même. C'est donc une identification en trompe-l'œil. Un toponyme, lui, peut ouvrir sur un temps originel en évoquant une fondation mythique, l'abolition d'une mauvaise coutume etc. et s'avère en général plus parlant que l'anthroponymie. Au terme de cette partie fort intéressante, Florence Plet constate toutefois avec réalisme que «l'onomastique romanesque remplit sa fonction classificatoire de façon assez sauvage» (p. 369) et que l'on est loin de certains systèmes décrits par les anthropologues, où chaque nom révèle la position qu'occupe son porteur dans la société. Tout au plus peut-on revendiquer l'existence de constantes génériques immuables qui empêchent des porteurs de «noms épiques» de passer dans un roman arthurien ou vice versa. Mais Florence Plet a aussi raison quand elle écrit qu'il faut tenter de déchiffrer ces données du Tristan en prose puisqu'on a affaire, indubitablement, à un «vaste document d'anthroponymie littéraire» (p. 370) et j'estime, pour ma part, que ce sont là les pages les plus réussies de son livre. Même si le Grand Système n'a pas pu en émerger, nous savons au moins pourquoi. C'est parce que - tel est l'enseignement du travail de F. Plet - il n'existe pas de Grand Système: il existe, par contre, des règles visibles et valables pour certains pans, comme le constat que l'insertion dans un lignage ne se fait qu'exceptionnellement par le rappel du nom de la mère mais assez couramment par celui de l'oncle maternel, une filiation matrilinéaire peut donc tout à fait s'afficher. A un niveau plus proprement linguistique, on apprend que les titres, lors de la désignation par autrui, n'interviennent pas de la même façon que pour l'autodésignation: aucun personnage ne se réfère à lui-même en utilisant un titre comme 
Messire, très fréquent, par contre, quand on s'adresse à quelqu'un d'autre. Ce sont là autant d'acquis dans le domaine de nos connaissances du roman arthurien en prose. Mais on comprend que F. Plet ait voulu aller plus loin et tenter, dans un dernier mouvement (Le Pouvoir des Noms, pp. 371-469), de s'interroger sur les motivations des noms. Tristan, par exemple, porte ainsi en lui tout son destin, et l'on peut donc suivre à la trace ce que «signifie» le nom. Pour d'autres personnages, c'est moins évident, et F. Plet doit donc se livrer à un déchiffrage plus spéculatif de quelques noms propres pour tenter de reconstituer le jeu des associations et connotations qu'un public médiéval pouvait ressentir au moment d'entendre des noms comme Le Morholt, Yseut, etc. Le dernier chapitre s'intitule d'ailleurs Gloses aventureuses et propose des analyses «sémantiques» de Taulas de la Montagne, Lucanor et quelques autres géants, mais aussi du Castel Marin, que le Tristan en prose motive de façon plus surprenante, Louveserp et Loviglai et d'autres. De par leur nature, ces pages sont un peu plus spéculatives que le reste du livre et s'assument comme telles. Il serait aussi facile que vain de proposer d'autres itinéraires d'associations à leur place, mais on comprend qu'il les fallait au livre. Une bibliographie, très complète du côté littéraire, ainsi qu'une série d'index, peut-être trop élaborée pour le lecteur pressé, complètent le volume.

Le présent livre est issu d'une thèse de Paris X soutenue en 2000, et le temps qui s'est écoulé, remarquablement long par rapport à d'autres publications de la même collection, entre l'achèvement de la thèse et la parution du volume, a été utilement mis à profit pour produire un vrai livre. Il reste, certes, un certain nombre de francocentrismes, comme la conviction que «l'étymologie», qui rattache Tristan à triste «ne fonctionne qu'en français» (p. 78) ou l'idée que depuis une thèse non publiée de Paris X de 1998 «nous disposons d'une terminologie précise» (p.257) pour parler des désignatifs, qui laissent un peu dubitatifs les lecteurs en dehors de l'Hexagone. Ces derniers peuvent aussi ne pas comprendre pourquoi l'auteur estime qu'il n'est pas nécessaire d'indiquer, dans la bibliographie d'un ouvrage scientifique, les pages pour des articles parus en volume ou en revue. Mais le plus important est que Florence Plet conduit son lecteur avec pédagogie et doigté à travers les diverses étapes de sa réflexion. Dans la mesure où l'échantillon des noms propres reste le même du début à la fin, le lecteur a toutefois parfois le sentiment de repasser par des lieux qu'il a déjà visités en compagnie de personnages qu'il a déjà vus: ainsi, il est question de géants dans le contexte de la toponymie, où ils commémorent un passé désormais révolu (pp. 357-361), et, plus tard (pp. 428-440), lorsque sont analysés leurs noms. Certaines citations, comme celle de Roland Barthes, utilisée p. 99 et puis p. 245, n'en deviennent pas plus claires pour autant. On peut aussi être un peu déçu par la reprise sans contrôle d'informations partiellement vieillies (les répertoires de noms propres de West, largement tributaires des travaux de Löseth et Sommer pour le domaine des romans en prose, seraient à vérifier sur la base des éditions modernes) ou hasardeuses (ainsi, p.107, on apprend que le sens de conjointure est «quelque chose qui transforme un ensemble amorphe en un tout organique» d'après E. Vinaver), mais les authentiques bévues sont rares: p. 121 la citation de P. Zumthor me paraît dire le contraire ou le corollaire de ce que veut dire F. Plet; p. 343-344 une explication possible - et plausible de la genèse du personnage de Tor, fils d'Arés, se lit dans le répertoire de West, que $\mathrm{F}$. Plet consulte souvent, pourtant; p. 386 le sens de «sauvage» du mot obscure me semble secondaire et métaphorique et ne devrait pas être mobilisé pour l'argumentation en question; p. 394 legier, dans le nom Acorant le Legier, ne veut pas dire «de petit prix», «insignifiant», mais «agile» et vient renforcer Acorant, qui, lui, n'a rien à voir avec 
«tuer». C'est donc un nom propre parfaitement cohérent, sauf si on applique au roman une esthétique post-moderne. Mais ces détails ne pèsent pas bien lourd face aux apports du travail de Florence Plet qui ouvre à presque toutes les pages d'intéressantes pistes pour des recherches futures, surtout si on consultait les manuscrits: p. 347 elle note à juste titre un air de famille qui s'installe à travers la sonorité dans la fratrie des fils de Loth: Gauvain, Agravain, Gaheriet, Guerrehet. Or, il se trouve que cette proximité phonique et graphique a conduit certains scribes à confondre les deux derniers, comme si, justement, ils étaient tellement proches qu'on pouvait substituer l'un par l'autre, abolissant, du coup, l'individualité que confère le nom propre. Cette plongée qui aurait conduit Florence Plet des éditions à la réalité des manuscrits aurait ajouté une dimension supplémentaire à son travail, il ne lui incombait pas de faire cette descente. Grâce à son livre, d'autres sont maintenant armés pour faire ce voyage. 